\title{
Improvements in Three-Dimensional Compositional Analysis of Complex Alloys
}

\author{
R. M. Ulfig*, E. Oltman*, D. J. Larson,* and V. S. Smentkowski** \\ * Imago Scientific Instruments Corporation, 5500 Nobel Drive, Madison, WI 53711 \\ ** General Electric Research, 1 Research Circle, Niskayuna, NY 12309
}

The performance of many alloys is limited by the complex three-dimensional (3D) nanoscale features and their impact on important properties like strength, toughness, and ductility. Atom probe tomography (APT), transmission electron tomography (TEM), and secondary ion mass spectrometry (SIMS) have been demonstrated to be useful tools to monitor complex nano-structural evolution in many materials systems, but in the past have not been employed to analyze these alloys with high spatial resolution, high mass resolving power, high analytical sensitivity, and adequate field of view (FOV) simultaneously [1,2]. With the advent of the high-speed, wide FOV atom probe instruments, some of these previous challenges were met, but direct peak overlaps and limitations of the mass resolving power can still constrain comprehensive analysis of complex alloy materials that contain many elements.

With recent performance enhancements in mass resolving power, and new implementation of peak decomposition and deconvolution software [3], accurate quantification and chemical identification over nano-scale and near-micro-scale features can be realized. The development of large angle reflectron technology for atom probes is responsible for the performance improvements and this technology has been incorporated into commercial instrumentation (LEAP $3000 \mathrm{HR}^{\mathrm{TM}}$ ). The current work shows the benefits of the performance improvements for measurements made on nano-featured nickel-base superalloy materials, for example, the spectral improvements and resultant nano-scale compositional sensitivity illustrated in Fig. 1.

The LEAP $3000 \mathrm{HR}$ instrument provides data with symmetric mass histogram peaks, low background levels and mass resolving powers of over 1 part in 300 at full-width hundredth maximum with sub-nanometer spatial resolution. Not only does the mass resolving power allow one to confidently separate peaks like $\mathrm{Ni}^{2+}$ and $\mathrm{Mo}^{3+}$, which are less than $1 / 6^{\text {th }}$ of a Dalton apart as shown in Fig. 1, it can allow confident identification of a peak of oxygen from that of sulfur $(<0.009 \mathrm{Da}$ separation) in data, even when these species are present as the minority species, as shown in Fig. 2. Where direct peak overlaps exist, the high mass resolving power and optimized peak shapes allow direct implementation of peak deconvolution techniques to maximize compositional accuracy [4]. In this analysis, overall Mo composition was measured as 4.5 at $\%$ with peak assignments ignoring direct overlaps, and 4.9 at $\%$ when using peak decomposition techniques. The results include the expected isotopic distribution and a nearly $10 \%$ improvement in compositional accuracy for this element. Such instrument performance and resultant data is of critical importance for the analysis and performance improvements of industrially important multi-component metallic alloy samples containing species such as $\mathrm{V}, \mathrm{Cr}, \mathrm{Fe}, \mathrm{Co}, \mathrm{Si}, \mathrm{N}, \mathrm{Cu}, \mathrm{Mo}$ and/or $\mathrm{Ni}$ and thus have significant overlaps in the mass histogram.

[1] C.T. Sims, et al., Superalloys II, High Temperature Materials for Aerospace and Industrial Power. (1987) John Wiley and Sons.

[2] B. Deconihout, et al., Applied Surface Science 76/77 (1994) 145.

[3] A. Cerezo, et al., Microscopy and Microanalysis 13 (2007) 408.

[4] M.K. Miller, et al., Atom Probe Field Ion Microscopy. (1996) Oxford University Press. 

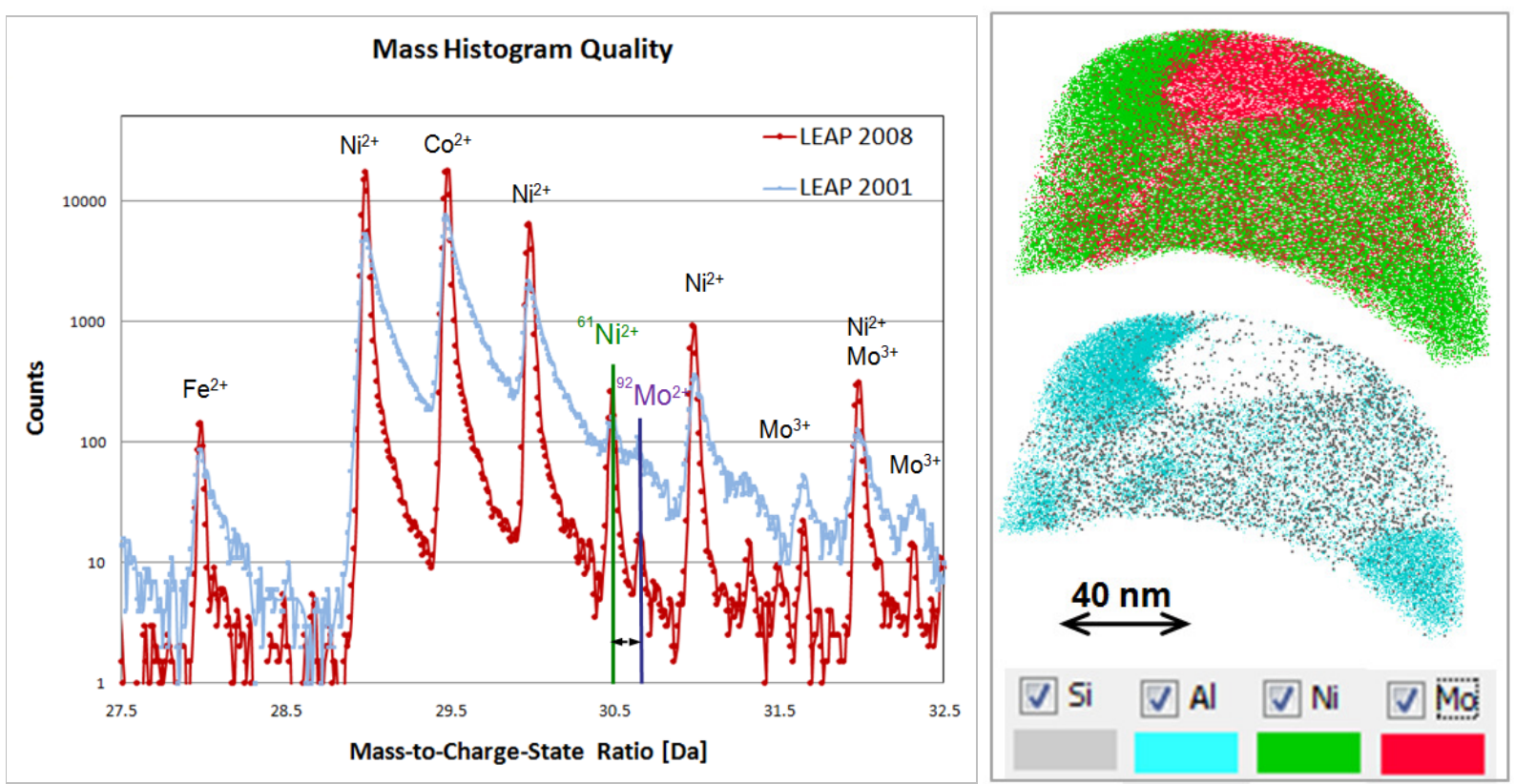

FIG. 1. Log scale mass histogram improvements for voltage pulsed LEAP systems on Ni-based superalloy[Left] and the spatial distribution of $\mathrm{Si}, \mathrm{Al}, \mathrm{Ni}$, Mo in the dataset for the high resolution data from a LEAP 3000 HR system [Right] (only a fraction of the atoms plotted for clarity)

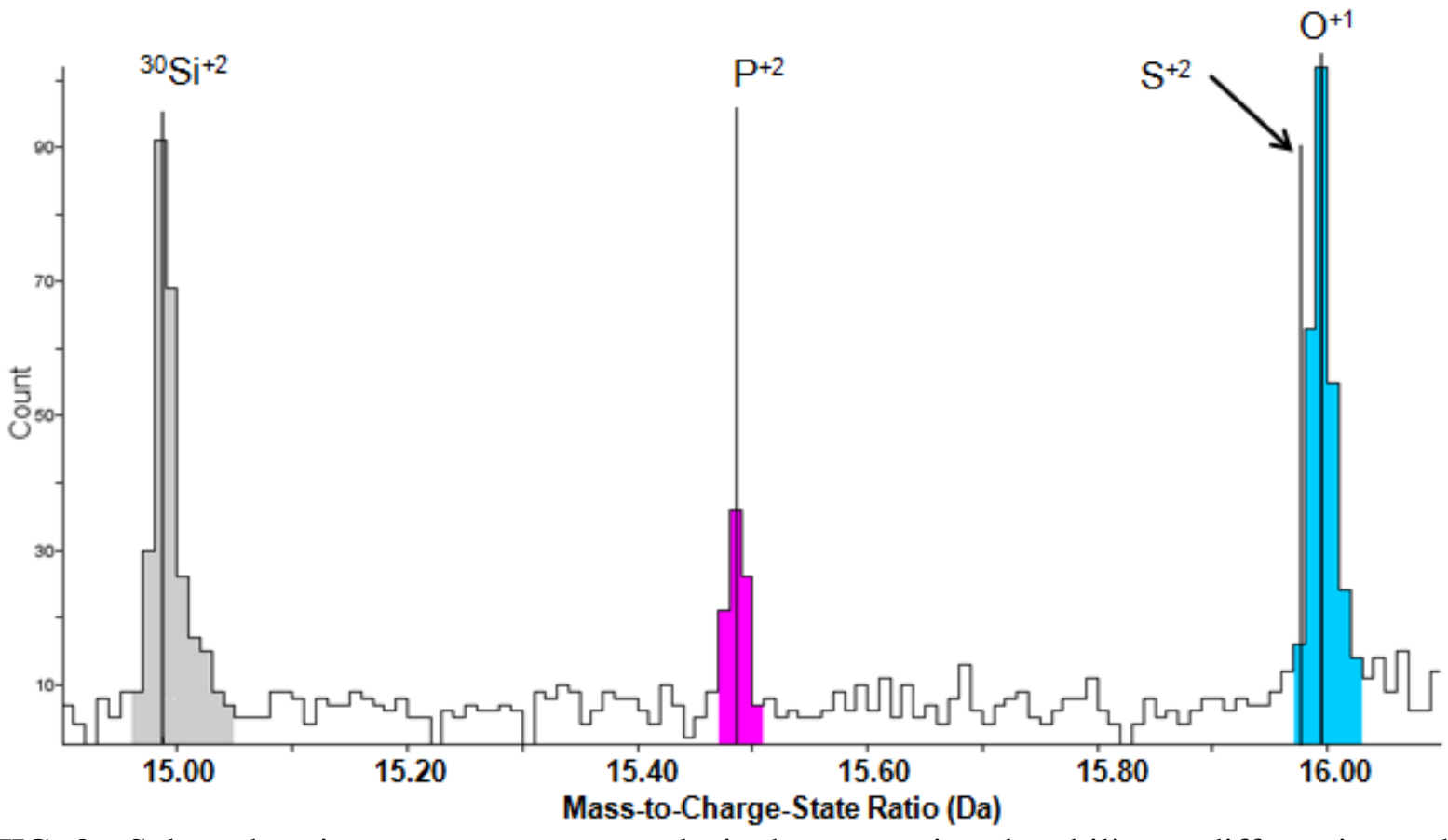

FIG. 2. Selected region mass spectrum analysis demonstrating the ability to differentiate sulfur and oxygen due to very high precision in the mass-to-charge-state ratio. Note the clear alignment of the phosphorus peak with the expected position and the same alignment of the peak at approximately 16 Dalton with the expected position of oxygen clearly different than if the species had been sulfur. 